\title{
Humidifier Device
}

National Cancer Institute

\section{Source}

National Cancer Institute. Humidifier Device. NCI Thesaurus. Code C49992.

A device designed to add moisture to the air. 